\section{Beyond the current database}

The database of alternatives will need to be updated continually with changes that occur as the result of regulations and their interpretation. However, even in its current preliminary state, it serves several important uses. The database can now be used to identify crop and pest situations in critical need of research. It can also be used to generate preliminary assessments of the economic impact of specific control practices or of restrictions on their use.

Information in the database must be applied carefully, however, because the alternative control strategy that will ultimately be used and even its degree of universal availability may not be readily apparent. Cost and benefit considerations will affect the individual grower's choice of control strategy. We can assume that the grower will choose options that will maintain his or her competitive advantage with other growers. The analysis becomes more complex when social and environmental factors are included. Even when regulations restrict the options of all growers, and assuming that some of the restrictions would influence the cost, quality, or availability of some products, market forces will undoubtedly continue to influence what growers can produce and at what production level. Consumers of raw agricultural products, including packers and processors as well as the general public, will help to clarify decisions for growers based upon their reaction to the changes they perceive in the cost or quality of products. This might result in changes to crop production systems that we cannot predict accurately at this time.

Frank G. Zalom is Director, Statewide IPM Project, IPM Implementation Group, and Extension Entomologist, based at UC Davis; and Joyce F. Strand is Computer Systems Manager, IPM Implementation Group, also at UC Davis.

The pesticide alternatives database discussed in this paper will be available in early August, 1990 . For ordering information, write or call: Statewide IPM Project, IPM/IG, University of California, Davis, California 95616, telephone (916) 752-8350.

\title{
The research imperatives: knowledge to reduce the use of broadly toxic pesticides
}

\author{
Mary Louise Flint
}

The University of California has been a leader in the development of methods to reduce reliance on pesticides. California was the site of the first major successes of biological control for insect pests during the latter part of the 19th century, and the term integrated control (the forerunner of integrated pest management) was coined by UC entomologists in the late 1950s.

California has continued as a leader in innovative pest management, making pioneering contributions in the areas of genetic improvement of crop plants and natural enemies, microbial control, cover crops, cultural controls, use of pheromones, and selective use of pesticides within integrated pest management (IPM) programs. As Zalom and Strand show in the preceding paper, this research has resulted in many successful applications. However, much more progress would be required to eliminate all need for the pesticides targeted by the California Environmental Protection Act of 1990 (EPA 1990) and the ongoing FIFRA reregistration process.

Most pesticide alternatives are very specific in their action, so they must be developed individually for each pest and crop situation. For instance, most biological control agents are effective only against one pest or a small group of pests. The narrow range of affected organisms is part of what makes these techniques more environmentally sound than broad-spectrum pesticides, but it also accounts for their limited development. The grower normally has a dozen or more major pests to contend with in any given crop; providing an alternative for just one pest won't be very helpful unless it is part of an IPM program that considers appropriate, nondisruptive ways to manage the other pests as well.

This points to the need to develop ecologically based, crop-oriented IPM programs that will become part of the overall crop or animal production system. Few of these techniques will be adopted unless research and extension workers can adapt and disseminate them in appropriate ways and demonstrate their economic viability.

The discussion below outlines the biological, cultural, chemical, and issue-related areas of research that are likely to lead to a reduction in the use of broad-spectrum pesticides. After a brief description of each area, its critical research needs are listed. Substantial expertise in many of these areas already exists at the University of California and elsewhere, and much research is in progress. How- ever, these efforts will have to accelerate in order to bring techniques rapidly to the field and broaden their application.

\section{Biological options}

- Classical biological control involves the deliberate introduction and establishment of natural enemies where they have not previously occurred. Most classical biological control programs target pests of exotic origin that have accidentally been introduced into new areas, and become established in the absence of their key natural enemies. Often, introduction of a well-adapted natural enemy can bring the pest under such complete control that it requires no further pest management action. This technique has proven successful against many insects and weeds.

Critical research needs: better understanding of the ecology of target pests and their natural enemies under the full range of climatic conditions in California; more foreign exploration; better facilities for rearing imported beneficials; better techniques for evaluating success

- Augmentative release of natural enemies involves the mass rearing of natural enemies in a rearing facility, and their subsequent release in pest-infested fields. Unlike classical biological control, this technique generally requires the periodic release of large numbers of natural enemies for adequate pest management. Predaceous or parasitic insects, mites, and nematodes have been used in this manner. Microbial agents are covered below.

Critical research needs: better rearing techniques; development of artificial host media; better release techniques; knowledge of timing of releases

- Cover crops and living mulches are resident or planted noncrop species grown on an orchard floor or on fallow land to reduce undesirable pest populations or provide other benefits. When used as mulches (either living or killed), they allow annual crops to be planted with nontillage or reduced tillage. Weeds may be suppressed through physical competition or allelopathy. Nematodes may be suppressed through antagonistic or allelopathic effects, or by preferential attraction to the cover crop (also called trap crop) over the desired crop. Cover crops also may provide habitat for natural enemies of insects, mites, and other pests.

Critical research needs: more knowledge about the impact of cover 
crops on weed, insect, pathogen, and nematode pests and crop growth, and the mechanisms involved in pest control (e.g., allelopathy, light exclusion, antagonism, etc.); more testing of potential cover crops, trap crops, and living mulches

- Genetic improvement of natural enemies can allow natural enemies that have proven successful in some environments to be made useful in other environments. Genetic improvement could include the modification of genes so the natural enemy could survive certain pesticide treatments or harsher climatic conditions. Natural enemies might also be selected to attack other pest species.

Critical research needs: better rearing methods for natural enemies; better understanding of the genetics of natural enemies; development of genetic markers to evaluate the effectiveness of newly released strains

- Microbial agents include bacteria, fungi, viruses, protozoa, and nematodes that are pathogenic or antagonistic to pest species, including insects, plant pathogens, and weeds. Some agents may occur naturally in the field, but most of the work and promise of research is directed at agents that can be mass produced and applied as pesticides. Because these agents can be chosen to be selective, killing only certain groups of pests, they may be much safer for humans and wildlife than conventional pesticides. Because their specificity reduces the size of their potential market, however, companies are also less likely to pay for their development.

Critical research needs: basic research to explore for additional microbial agents; application technology and methods or formulations to extend the pathogens' viability in the field and on the shelf; more biological/ecological information to allow more effective placement and timing of application; incentives for pesticide companies to invest in these limited-market materials; further selection and genetic engineering work to extend the host range of promising microbials

- Host plant resistance involves the development of crop or ornamental varieties that are able to resist or tolerate pest attacks that would kill or seriously damage other plants of the same species. In the case of weed pests, resistant cultivars would have an improved ability to compete with weeds, either through physical or allelopathic means. Once developed, host plant resistance provides a very inexpensive, environmentally sound, and highly effective way to avoid pest problems.

Critical research needs: expansion of biotechnological methods of developing resistant cultivars; more research into the mechanisms of resistance to determine a pest's ability to overcome host plant resistance; regular pre-release screening of all new varieties for pest resistance

- Management of natural enemies can often improve the effectiveness of native natural enemies by altering the way a crop is grown or modifying adjacent areas to provide improved shelter or food for natural enemies. For example, planting a strip of alfalfa in a cotton field can improve biological control of cotton pests.

Critical research needs: better biological information about natural enemies to determine food and shelter requirements and dispersal habits; development of attractants for natural enemy species (e.g., wheast, pheromones)

- Autocidal controls are tactics that cause the pest to contribute to the reduction of its own population. The primary method is to release enough sterile insects of a species to effectively reduce the chances of a wild female mating with a fertile male. Under ideal conditions, repeated releases of sterile insects can eliminate an invading pest species. Another approach is to develop and release strains of a pest that will either reduce the species' overall fitness or introduce harmful genetic traits.

Critical research needs: better methods of rearing sterile insects so they can remain competitive with native populations; less expensive methods for sterilization and mass rearing

\section{Cultural options}

- Crop rotation involves the deliberate planting of specific crop sequences to make the year-to-year survival of soil-dwelling pests difficult or impossible. Crop rotation can provide good control for certain fairly host-specific soilborne plant pathogens and nematodes, and can also contribute to weed management. Only a few insect pests are good candidates for this method.

Critical research needs: determination of alternate crops and lengths of rotation required to manage pests and reduce weed seed banks under different cropping systems and environments; integrate new pest-resistant cultivars into rotations; re-evaluate economic feasibility of rotations

- Physical control methods include the mechanical destruction of pests or creation of physical barriers to pest infestation or movement. A wide variety of methods could be included in this category - some are regular farm practices, such as cultivation; others are practiced especially for pest control, such as solarization, artificial mulches, row covers, sticky barriers, or "bug vacs."

Critical research needs: reduction of the labor-or material-intensive nature of many of these methods; economic analysis and development of new products and ideas to make their use more viable - Sanitation is any practice that removes overwintering refuges or alternate refuges where pests can survive when the crop is not in the field. Sanitation also includes equipment cleaning. Many of these methods are just plain "good farming practices," such as weed removal and the rapid removal of crop debris; however, research can provide more efficient and effective methods.

Critical research needs: more efficient and effective methods; better understanding of pest dispersal, life cycles, and overwintering

- Irrigation management techniques can increase or decrease the damage brought on by insects, mites, or plant pathogens, and affect weed competitiveness. Response varies with pest and crop.

Critical research needs: consideration of altered irrigation practices from a crop-pest ecosystem perspective; better understanding of the broad effects of changes in water application: flood, furrow, sprinkler, or drip

- Pruning and canopy management can sometimes affect a tree or vine's habitability for pests. Some pruning practices may make other management methods more feasible (e.g., biological control, pheromone confusants, pesticide application, or sanitation).

Critical research needs: better knowledge of pest distribution in plant canopies and the microclimatic alteration possible through pruning; additional studies on the extent of photosynthetic area that can be removed without significantly affecting plant health in the long or short term

- Strategic choices take in all of the decisions growers make at planting time, including the choice of field (soil type, previous crops, existing pest populations, etc.), location of the field (e.g., relative to sources of pest infestation or inoculum), and time of planting (e.g., relative to major pest migrations or favorable weather conditions).

Critical research needs: better information on pest biology (dispersal, overwintering, and agroecosystem relationships would provide leads in this area)

- Regulatory management practices, as applied here, includes any practice that must be instituted area-wide in order to be effective. This might include mandatory host-free periods, host-free zones, crop termination dates, or limits on the cultivars or seeds that can be planted. Usually these practices must be enforced by law or by grower groups.

Critical research needs: better understanding of the socio-economic-environmental benefits that will motivate growers to adopt these practices

\section{Chemical options}

- Synthetic organic pesticides make up most of the pesticides in current use. They kill pests through a variety of mechanisms. Many of these materials have harmful effects on nontarget organisms, including people, because of their chemical properties and broad toxicity.

Critical research needs: development of pesticides that are more 
environmentally safe; better ways to evaluate pesticide impacts in the environment and on human health; improved application technology

- Oils, soaps, and other fatty acids are insecticides and acaricides that can be effective, but have less impact on human health and wildlife than synthetic organic chemicals when properly applied. Fungicidal and herbicidal soaps also have potential.

Critical research needs: determination of phytotoxicity; improvement of application technology

- Botanical insecticides are extracted or derived directly from plants. Common botanicals include pyrethrin, neem, rotenone, sabadilla, ryania, and nicotine. Some of these materials have fairly broad toxicity, but because they break down rapidly in the environment, many people consider them much safer than synthetic organic materials.

Critical research needs: find new ways to use existing materials, new application technologies, and new formulations; search for new plant compounds for pest control

- Semiochemicals, including allomones, kairomones, and pheromones are substances given off by plants or animals that cause specific behavioral responses in other individuals. Allomones and kairomones are given off by individuals of one species to affect another species; allomones (such as repellents, deterrents, or antibiotics) favor the producer, and kairomones (such as attractants or feeding stimulants) favor the receiving organism. Either type could be useful for pest management. Pheromones are given off by individuals of a species and elicit a specific behavioral response in other individuals of the same species. The most common pheromones used in pest management are sex attractants that attract individuals of one sex for mating. Other types, such as trail pheromones for ants and aggregation pheromones for bark beetles and other insects, have potential for pest management.

Critical research needs: identification of additional semiochemicals; better substrates for releasing semiochemicals into the environment; better application technology; combinations of semiochemicals that could enhance effectiveness

- Pesticide application technology could be improved in a variety of ways to reduce the volume of pesticide use and make it substantially safer. For instance, electrostatic applicators could place the pesticide material directly on the target plant, eliminating drift.

Critical research needs: better application equipment to limit the movement of material away from target areas, including increased use of electronics; development of better methods to treat limited parts of plants or rows; expansion and improvement of low-volume application techniques; improved formulations and application techniques to make environmentally sound materials effective in more situations

- Treatment thresholds (control action guidelines) and sampling methods provide a way to decide whether management actions, including pesticide applications, are needed to prevent eventual losses from pest damage. Using various sampling methods, researchers or pešt managers can get an accurate estimate of the pest populations in the field and correlate that with the potential for damage. Treatment thresholds and sampling methods allow growers to avoid extra pesticide applications by giving them a clear idea of the specific pest potential.

Critical research needs: better understanding of the relationship of pest populations to yield and quality; integration of natural enemy populations into thresholds; simpler and more accurate sampling methods and monitoring devices; better and more reliable attractants for traps; basic pest biological research to determine where (e.g., what plant part) and when (e.g., what pest stage, what time of day or season) is best for monitoring certain pests

\section{Related education, economic, and policy issues}

- Education techniques for information on new pest management methods and ways to reduce pesticide use need to be rapid and effective. If not adapted properly to grower needs and presented understandably, even the most promising alternatives will not be adopted by growers.

Critical research needs: investigations of how pest management information is most effectively communicated in the grower community under current pressures and constraints; evaluation and improvement of current delivery methods (e.g., publications, videos, computer programs, demonstrations, workshops, or test plots); investigation of new techniques

- Economic comparisons can establish the comparative costs of alternative pest control techniques under current and future pricing structures. For growers, such comparisons are prerequisites for the adoption and implementation of new practices.

Critical research needs: comparative studies of pest management alternatives in a broad array of cropping and marketing systems throughout California

- Policy issues involve many of the constraints that limit growers' adoption of new pest management or production systems. These may include a grower's unwillingness to assume a perceived risk, a financial institution's unwillingness to support untested systems, and public agricultural and environmental regulatory policies.

Critical research needs: research and analysis of various policies that may limit the adoption of new technologies; analysis of the potential for "crop insurance" to assume a portion of the risk associated with the uncertainties of alternative systems; survey financial institutions regarding their willingness to accept risks associated with new or different pest management technologies; review regulatory policies that may impact pest management, including registration procedures and the licensing of pest control professionals (e.g., potential conflicts of interest)

\section{Summary and conclusions}

A directed research program on alternatives to the pesticides of current concern could yield a variety of new, environmentally sound pest management techniques as well as broaden the use of some currently available alternatives. Alternatives would include biological, cultural, and chemical methods. Some may become available rapidly; others may only be ready to be brought to the field after many years.

Many newly developed methods would at first be appropriate only for one or a few pests, probably in a limited-crop situation. Expanding their use to additional pests and crops would require further research. Many of the alternatives listed above are appropriate only for certain kinds of pests, and some new techniques may have undesirable environmental or health effects; all must be evaluated fully before their release. Future environmentally sound, sustainable pest management systems will require the integration of a number of techniques through an IPM program in order to provide reliable control and guard against the development of pest resistance.

The development of alternatives must be a cooperative effort of basic and applied researchers. New materials and techniques cannot be found without basic research, and they cannot be made useful or adoptable without the cooperation of applied researchers working in a range of crops and situations. Techniques must be made compatible with current production systems. Good dissemination methods are critical. No new technology will be adopted widely unless extension workers can show growers and pest control advisors that it can work on their farms and that it will be effective. In some cases, policy and economic constraints will have to be removed in order to foster the widespread use of these less-toxic alternatives.

Mary Louise Flint is Director, IPM Education and Publications for the UC Statewide IPM Project, and Extension Entomologist, Davis Campus. 